\begin{tabular}{|c|c|}
\hline Title & $\begin{array}{l}\text { Color-coded visual ization of microbubble clouds interacting with eddies in a spatially developing turbulent boundary } \\
\text { layer }\end{array}$ \\
\hline Author(s) & Park, Hyun Jin; Saito, Daichi; Tasaka, Y uji; Murai, Y uichi \\
\hline Citation & $\begin{array}{l}\text { Experimental thermal and fluid science, 109, UNSP } 109919 \\
\text { https://doi.org/10.1016/.expthermflusci.2019.109919 }\end{array}$ \\
\hline Issue Date & $2019-12$ \\
\hline Doc URL & http:/hdl.handle.net/2115/83367 \\
\hline Rights & $\begin{array}{l}\text { (9 2019. This manuscript version is made available under the CC-BY -NC-ND } 4.0 \text { license } \\
\text { http://reativecommons.org/icenses/by-nc-nd/4.0/ }\end{array}$ \\
\hline Rights(URL) & http://creativecommons.org/icenses/by-nc-nd/4.0/ \\
\hline Type & article (author version) \\
\hline File Information & ETFS2019_MB_Revised_New_HUSCAP.pdf \\
\hline
\end{tabular}

Instructions for use 


\title{
1 Color-coded visualization of mic robubble clouds interacting with 2 eddies in a spatially developing turbulent boundary layer
}

Hyun Jin Park*, Daichi Saito, Yuji Tasaka, Yuichi Murai

Laboratory for Flow Control, Faculty of Engineering, Hokkaido University, N13-W8, Kita-ku, Sapporo 0608628, Japan

* Corresponding author. Tel/fax: +81 11706 6373, E-mail address: park@eng.hokudai.ac.jp.

\section{Highlights}

- Turbulent eddy-microbubble interaction is investigated by two visualization methods.

- Microbubbles in a boundary layer are 3-dimensionally visualized by color illumination.

- These bubbles are accumulated in a vortical core by pressure gradient in the vortex.

- Also, streamwise vortices in a boundary layer are investigated by flake visualization method.

- Span-distance between vortices is increased by microbubbles injected into the layer.

\begin{abstract}
Three-dimensional distribution of microbubbles dispersed in a turbulent boundary layer spatially developing along a flat plate is investigated experimentally. The aim of study is to find out visually how microbubbles interact with turbulent eddies to achieve frictional drag reduction on a wall. Setting conditions of the experiments are stream Reynolds number up to $2.3 \times 10^{4}$, microbubble volume fraction inside boundary layer less than $10^{-5}$, and microbubble Stokes number smaller than $10^{-3}$. Stream-wise vortices in the boundary layer were visualized by flake optics, of which spacing expands with injection of microbubbles. 3-D microbubble distributions were visualized by a colorcoded volumetric illumination. The result showed preferentially accumulated microbubble clouds to low-speed streaks close to viscous sublayer and to hair-pin vortices in the buffer layer, leading to reduced diffusivity of microbubbles relative to the turbulent momentum diffusivity.
\end{abstract}

Keywords; Drag reduction, Microbubbles, Turbulent boundary layer, Flow visualization

\section{Introduction}

Microbubbles injected into turbulent boundary layers can reduce skin frictional drag acting on a solid surface. This is a known fact since 1973 as McCormick and Bhattacharyya [1] obtained a significant degree of drag reduction by generating microbubbles around a submerged hull in water flow. Legner [2] explained the reduction with shear-thickening trend of viscous sub-layer as microbubbles increase local effective viscosity. 
1 Marie [3] attributed the reduction to decrease of Reynolds shear stress in the buffer layer with their accumulations. Question is where microbubbles tend to be accumulated inside turbulent boundary layers. Since 1990, many investigators tried to solve this experimentally with changing bubble size [4-8]. In these papers, the term of "microbubbles" was used in their paper title as a qualitative sense while actual size of the bubbles was ranged from 0.1 to $3.0 \mathrm{~mm}$, called "milli-bubbles" but not in $\mu \mathrm{m}$ order. Such milli-bubbles in turbulent boundary layers of water show high bubble deformability in most applications [9]. Role of the deformability in shear stress field was reported by Tasaka et al. [10] for unsteady laminar flows, and by van Gils et al. [11] for high-speed turbulent flows. In these regime, Capillary number or Weber number characterizes the modification of shear stress field. Further large bubbles in Froude number dependence regime, formation of gas film and wettability sway the wall shear stress as reported by Park et al. [12] and Kitagawa et al. [13].

In contrast, bubbles smaller than $100 \mu \mathrm{m}$, i.e., actually $\mu \mathrm{m}$-order bubbles, keep spherical shape without deformability in water up to $8 \mathrm{~m} / \mathrm{s}$ in flow speed. In CFD studies, such tiny bubbles are approximated as point-source dispersions and called also "microbubbles" being distinguished from deformable bubbles. Ferrante and Elghobashi [14] conducted DNS on microbubble-laden wall turbulence and found that microbubbles pushed streamwise vortices away from the wall. One of unsolved issues is a role of generation of microbubble clouds. Once the clouds are formed, microbubbles cannot be directly simulated under the assumption of point source. Inside such a cloud, volume fraction and interfacial concentration rise sharply to modify local fluid properties such as density, viscosity and viscoelasticity $[15,16,17]$. The generation is promoted as the slip velocity of microbubbles to liquid phase is rather small. This is explained by comparison with milli-bubbles crossing over the coherent structures of turbulence. As Mathai et al. [18] reported, microbubbles induce preferential concentration in turbulence even at Stokes number is sufficiently low. In experimental approach, effect of microbubbles on turbulent shears was reported by many researchers [1924]. Their experiments observed a high sensitivity to drag reduction or suppression of turbulence even in low bulk volume fraction of microbubbles. A key to explain the mechanism is to clarify where and how such microbubbles form clouds inside the boundary layers.

In the present experimental study, first, we show how the spatial distribution of coherent structure is altered with mixing of microbubbles. And then we investigate spatial distribution of microbubbles in the same situation. For the microbubble distribution inside the turbulent boundary layer, we have employed a three-dimensional (3-D) visualizing method. This method relies on volumetric illumination of microbubbles using a color-coded light projected in a wall proximity. The optical set-up requires only a single camera and it allows effective measurement volume to be kept large enough covering the whole boundary layer thickness. Similar technique was reported by Watamura et al. [24] and Xiong et al. [25] for tracer particles as a 3-D particle tracking velocimetry. With this method, it is presented how microbubbles form specific distributions during their two-way interactions between microbubbles and inner structures of wall turbulence. 


\section{Experimental Method}

\section{2-1. Experimental facility}

A schematic diagram of experimental facility is shown in Fig. 1. It consists of an open water horizontal channel of $3,485 \mathrm{~mm}$ in total length, a test plate set vertically in the downstream region of the channel, and recirculation units including a pump, a flowmeter, and a bubble separating reservoir tank. The open channel around the test plate is made of transparent acrylic resin for all the three sides to allow various optical visualizations. And it is covered with opaque shading curtains to block light from outside.

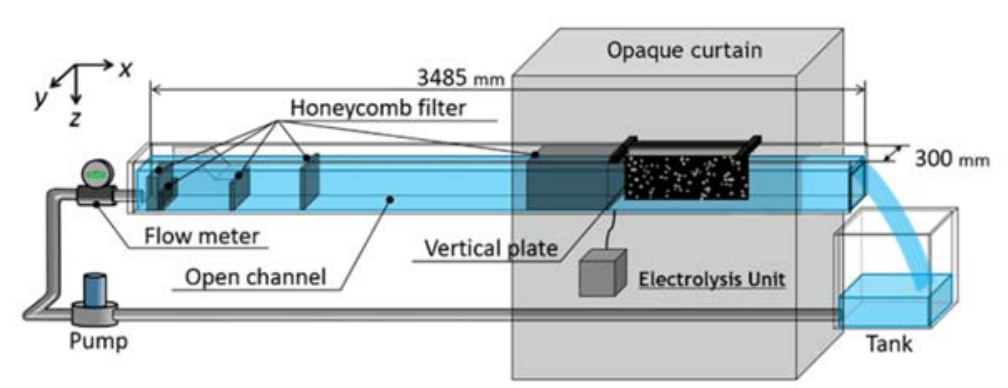

Fig. 1 Open channel facility for visualizing flat-plate turbulent boundary layer with microbubbles

The test plate is made of dielectric resin, which is $670 \mathrm{~mm}$ in stream-wise length, and $5 \mathrm{~mm}$ in thickness as depicted in Fig. 2a. During the experiment, the test plate is submerged into water flow at a controlled height (see Fig. 2b). Front of the plate is cut by $30^{\circ}$ at the backside of the flat surface forming a sharp edge to ensure parallel inflow as the starting point of a spatially developing boundary layer (see Fig. 2c). Four honeycomb filters are set inside the open channel to produce uniform inflow with sufficiently small turbulence intensity. The coordinate system is defined as the $x$ axis in the streamwise direction, the $y$ axis in the direction away from the plate surface, and the $z$ axis vertical.

a

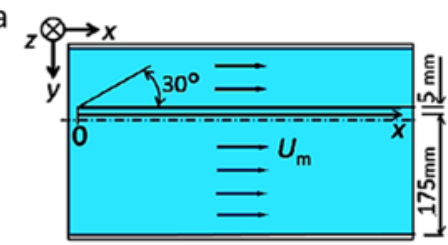

Top view

b

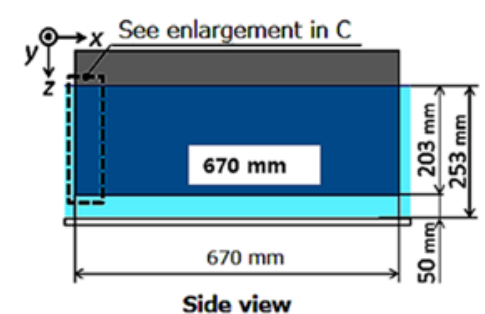

C

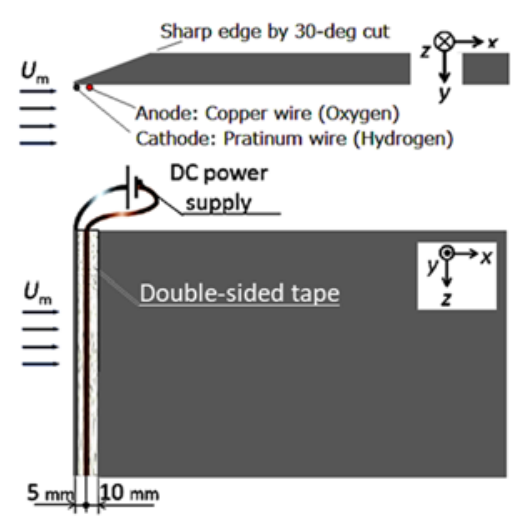

Fig. 2 Geometric set of wire electrodes at the front edge of a flat plate inserted in the open water channel;

(a) top view of the plate, (b) side view and (c) details of electrodes arrangement 
For generating microbubbles by water electrolysis, tap water of $0.5 \mathrm{~m}^{3}$ in total volume is used as liquid phase. At the front edge of the plate, a platinum wire of $0.50 \mathrm{~mm}$ in diameter as cathode and a copper wire of $0.45 \mathrm{~mm}$ in diameter as anode are attached in parallel to the $z$ axis with displacement of $5 \mathrm{~mm}$ (see Fig. 2c). Hydrogen gas released from the cathode as microbubbles is naturally conveyed into the boundary layer. Table 1 specifies the experimental conditions of microbubble generation. Diameter of the microbubbles $d$ is ranged from 10 to $50 \mu \mathrm{m}$, taking peak at $30 \mu \mathrm{m}$, which is much smaller than the boundary layer thickness, $O(10 \mathrm{~mm})$. The electrode wires also have a role of tripping wires to initiate perturbation leading flow transitions to ensure development of a turbulent boundary layer.

Table 1 Experimental conditions for microbubble generation

\begin{tabular}{lrl}
\hline Electric current $(J)$ & $0.08-0.10$ & {$[\mathrm{~A}]$} \\
Mean inflow velocity of water $\left(U_{\mathrm{m})}\right.$ & 67.1 & {$[\mathrm{~mm} / \mathrm{s}]$} \\
Temperature of water $(T)$ & $26.5-28.0$ & {$\left[{ }^{\circ} \mathrm{C}\right]$} \\
Kinematic viscosity of water $(v)$ & $0.839-0.868 \times 10^{-6}$ & {$\left[\mathrm{~m}^{2} / \mathrm{s}\right]$} \\
Environmental pressure $(P)$ & $101.3-102.2$ & {$[\mathrm{kPa}]$} \\
Diameter of hydrogen microbubble $(d)$ & $30 \pm 20$ & {$[\mu \mathrm{m}]$} \\
Flow rate of hydrogen bubble volume $\left(Q_{\mathrm{b}}\right)$ & $10.1-12.6 \times 10^{-9}$ & {$\left[\mathrm{~m}^{3} / \mathrm{s}\right]$} \\
Liquid volume flow rate inside boundary layer $\left(Q_{1}\right)$ & $0.172 \times 10^{-3}$ & {$\left[\mathrm{~m}^{3} / \mathrm{s}\right]$} \\
\hline
\end{tabular}

\section{2-2. Method of visualization}

Two different types of optical visualization are conducted to measure alteration of turbulent coherent structures and 3-D distributions of microbubbles as illustrated in Fig. 3.

For visualizng turbulent vortical structures in liquid phase, a flake aqueous solution (Kalliroscope AQ1000 , Kalliroscope Ltd.) is mixed into water. The flakes have $30 \times 6 \times 0.07 \mu \mathrm{m}^{3}$ in typical size, transparent crystal structure of 1.85 of refractive optical index, and $1.62 \times 10^{3} \mathrm{~kg} / \mathrm{m}^{3}$ in density [26]. They reflect light as like crushed mirrors dispsersed, and therefore, pararell incident light to the flow visualizes the spatial structure of turbulence [27]. This allows us to observe turbulent structure directly using the flakes without any PIV analysis. More details will be explained in Section 3-1. Amount of the solution mixed is $150 \mathrm{ml}$ in total corresponding to $300 \mathrm{ppm}$ in volume mixture rate (note that crystal solid volume fraction in the solution is 0.1 of this value). Up to this solition rate, non-Newtonian viscosity does not take place as shear rate is lower than $10^{2} \mathrm{~s}^{-1}$. As a source of light sheet, $532 \mathrm{~nm}$ continuous wave green laser with $3 \mathrm{~mm}$ in thickness is used to illuminate the flake in the vicinity of the plate as shown in Fig. 3a. A high-speed camera (CamRecord CR600x2, NAC Image Technology Inc.) set in front of the plate perpendicularlly recorded the visualized images at $500 \mathrm{fps}$. 
For visualizing microbubble clouds, a color-coded volumetric light is illuminated closely to the wall using 2 a liquid crystal display projector (LCDP) as shown in Fig. 3b. Images are recorded by a high-speed video camera (FASTCAM Mini AX-50, PHOTRON Ltd.) having color image sensors at frame rate of 500 fps. In almost cases of the visualization, the camera is set with right angle, $90^{\circ}$, to visualized plane. In our visualization, however, angle of optical axis of the volumetric light to the camera axis is set at $110^{\circ}$ as shown in the figure. Around this angle, between $110^{\circ}$ and $115^{\circ}$, it is possible to obtain brightest scattering image of microbubbles along Mie scattering principle of spherical bubbles [28].
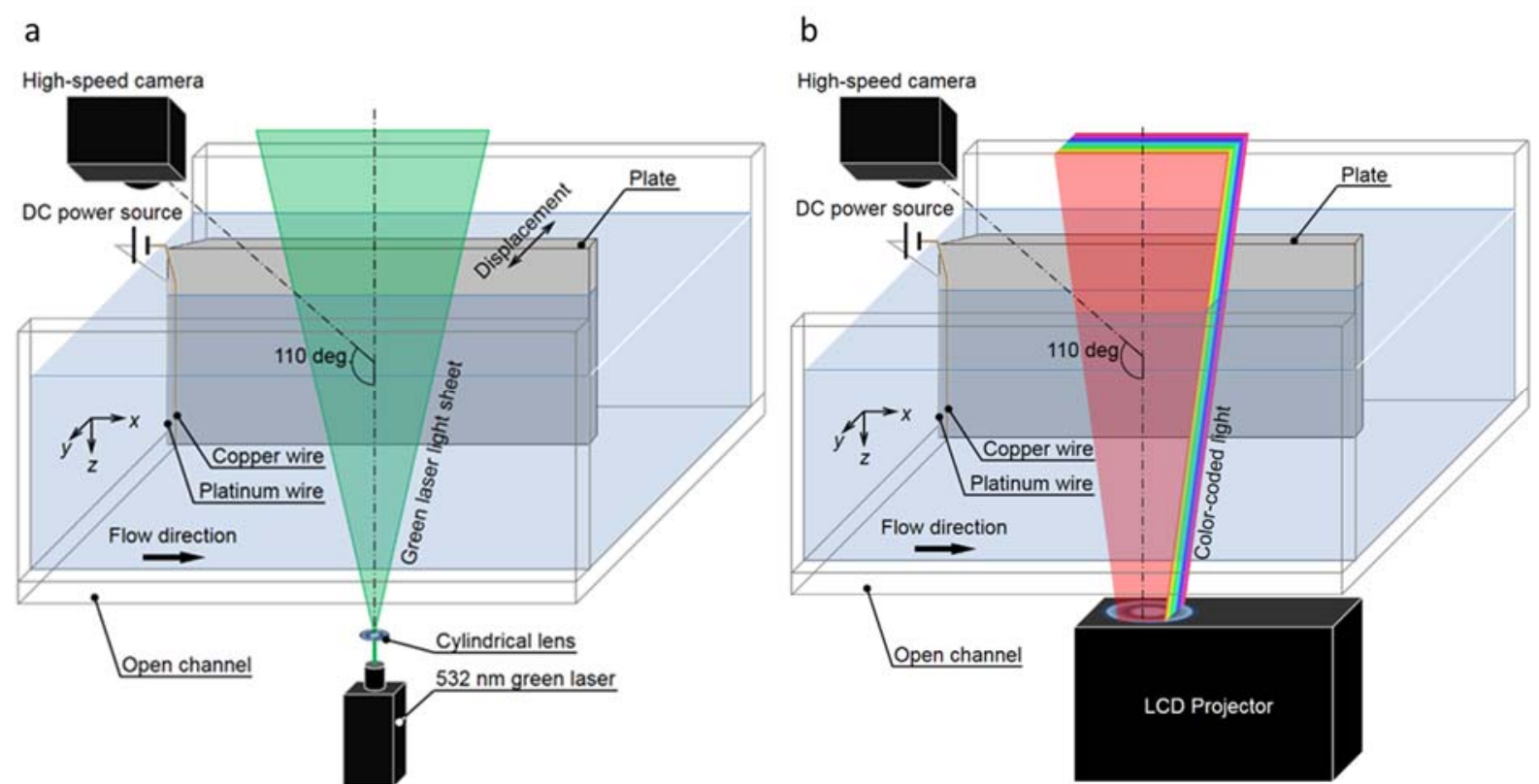

8

Fig. 3 Experimental setup for visualizing microbubble-laden flat-plate turbulent boundary layer in water;

(a) laser sheet illumination for visualizing turbulent vortical structures in liquid phase vicinity to the wall and (b) optical set-up for microbubble visualization using color-coded volumetric illumination

\section{2-3. Primary parameters in the experiments}

There are two kinds of Reynolds numbers which signify flow structures of the present boundary layer:

$$
\begin{aligned}
& R e_{x}=\frac{U_{\mathrm{m}} x}{v}, \\
& \operatorname{Re}_{\mathrm{f}}=\frac{u_{\tau} \delta}{v}, u_{\tau}=\sqrt{\frac{\tau_{w}}{\rho}},
\end{aligned}
$$

where $U_{\mathrm{m}}$ is mean inflow velocity of water to the test plate in the open channel, and $v$ is kinematic viscosity of water without microbubbles. The first definition, $R e_{x}$, termed stream Reynolds number, increases with the downstream distance, $x$. It takes the range up to $R e_{x}=2.3 \times 10^{4}$. $R e_{\mathrm{f}}$ in Eq. (2) defines frictional Reynolds number based on wall friction velocity, $l_{\tau}$, and boundary layer thickness, $\delta$, and it takes the range of $50<R e_{\mathrm{f}}$ 
$<150$ in this study, where $\tau_{\mathrm{w}}$ and $\rho$ are wall friction and density of water. Basically, these two Reynolds numbers are not independent of each other and show similar tendency because $U_{\mathrm{f}}$ and $\delta$ are increasing with $x$ in this situation.

For microbubble phase, following dimensionless parameters represent their status of motion:

$$
\begin{aligned}
& R e_{\mathrm{b}}=\frac{U_{\mathrm{b}} d}{v}, \\
& R e_{\mathrm{b}}^{\prime}=\frac{U_{\delta}^{\prime} d}{v}, \\
& C a=\frac{\mu \dot{\gamma} d}{\sigma} \square \frac{\mu d}{\sigma}\left(7 \frac{U_{\mathrm{m}}}{\delta}\right), \\
& S t=\frac{u_{\tau} d^{2}}{36 v l_{\tau}}=\frac{\tau_{\mathrm{w}} d^{2}}{36 v^{2} \rho}, l_{\tau}=\frac{v}{u_{\tau}},
\end{aligned}
$$

where $\mu, \dot{\gamma}, \sigma, d, U_{\mathrm{b}}, U_{\delta}^{\prime}$ and $l_{\tau}$ denote viscosity of water, liquid shear rate of surrounding bubbles, interfacial tension of bubbles, mean bubble diameter, free rising velocity of a single microbubble in quiescent water, velocity fluctuation of flow in the boundary layer and wall unit, respectively. In the experiment, microbubble Reynolds number with $U_{\mathrm{b}}, R e_{\mathrm{b}}$ in Eq. (3), takes the range of $0.1<R e_{\mathrm{b}}<1$, i.e. in Stokes to Oseen regime. Eq. (4) represents microbubble Reynolds number defined by the boundary layer velocity fluctuation $U_{\mathrm{b}}^{\prime}$, which is $R e^{\prime} \mathrm{b} \leq 0.1$. $C a$ in Eq. (5) defines microbubble Capillary number which is $C a<10^{-4}$ in the present condition, ensuring that microbubbles keep spherical shape. Here representative shear rate is given by liquid shear rate on the wall obeying 1/7 power law as an estimate. St in Eq. (6) stands for microbubble Stokes number which estimates the ratio of inertia response times of microbubble and of liquid phase in the boundary layer. In the experiment, it is always in $S t \leq 0.001$.

As a representative definition of volume fraction of the microbubble inside boundary layer, following estimation is used:

$$
\alpha=\frac{Q_{\mathrm{b}}}{Q_{\mathrm{b}}+Q_{1}}, Q_{\mathrm{b}}=\frac{J R T}{2 F P}, Q_{1}=W \int_{0}^{\delta} \bar{u}(y) d y \square \frac{7}{8} W \delta U_{\mathrm{m}},
$$

where volume flow rate of the microbubbles $Q_{\mathrm{b}}$ is given by Faraday law of water electrolysis: $J, R, T, F$ and $P$ represent electric current, gas constant, temperature, Faraday constant, and pressure inside the gas phase, respectively. Flow rate of the liquid phase inside the boundary layer $Q_{1}$ is estimated by the $1 / 7$ power law about the velocity profiles, where $W$ is the span-wise length of the plate. Present experiment takes the rage of $\alpha<0.01 \%$ (note that local volume fraction takes 2-3 digits higher value as microbubble clouds are formed). We also note that wall unit takes the range of $50 \mu \mathrm{m}<l_{\tau}<200 \mu \mathrm{m}$ in our experiment. Thus, diameter of the microbubbles, $30 \mu \mathrm{m}$, is comparable with or smaller than the wall unit in this study. 


\section{Alternation of turbulent eddies}

For high concentration of microbubbles in turbulent boundary layer, optical access such as PIV and LDV to the liquid velocity profile measurement is severely restricted. An alternative way to approach the liquid phase is flake optics that can directly visualize coherent structures in liquid phase. According to the previous studies [29-31], relationship between mean span-wise spacing of low-speed streaks $\bar{\Lambda}$ and the wall shear stress $\tau_{\text {wall }}$ is represented as

$$
\bar{\Lambda}=a l_{\tau} \propto 1 / \sqrt{\tau_{\text {wall }}} \rightarrow \frac{\tau_{\text {wall }}}{\tau_{\text {wall }, 0}}=\left(\frac{\bar{\Lambda}}{\bar{\Lambda}_{0}}\right)^{-2},
$$

where $a$ is a dimensionless proportional constant to the wall unit $l_{\tau}$ ranged at $80<a<120$. Hence, measurement of $\bar{\Lambda}$ in two cases with and without microbubble injection allows us to estimate how the wall shear stress is modified.

\section{3-1. Flake optics test}

To visualize turbulent vortical structures, flake aqueous solution is mixed in the water channel (product name: Kalliroscope (AQ-1000)), which consists thin platelets made of Guanine $\left(\mathrm{C}_{5} \mathrm{H}_{5} \mathrm{~N}_{5} \mathrm{O}\right)$ in crystalized state. The thickness of the platelet is about $10 \mathrm{~nm}$ while the width is less than $10 \mu \mathrm{m}$ as shown in Fig. 4a. The optical characteristics of the flake are available in our previous papers by Murai et al. [19] and Ohkubo et al. [27]. As in shear flows, the platelets orient to the primary strain rate direction, and reflect light to mirror direction so that instantaneous spatial structure of turbulent boundary layer can be visualized. Fig. $\mathbf{4 b}$ is a schematic for explaining the optical principle applied for the turbulent boundary layer [32]. This set-up visualizes arrayed pair of stream-wise vortices and low-speed streaks as green separated bands in imaging plane. For ejection part, a thick reflection band appears just in front of the part. Inside sweep event, a thin reflection line appears in front of the sweep, asymmetrically. Park et al. [33] applied similar technique for a horizontal turbulent channel flows using aluminum flakes of larger than $100 \mu \mathrm{m}$ in size. We employ the technique using Kalliroscope so that flake does not disturb microbubble motions both in individual and clouded states.

A sample snapshot of image is shown in Fig. 4c, which is the case without microbubble injection. There can be seen a stripe pattern as expected. Thick and thin bands mixed in the image correspond to the arrangement of stream-wise vortices. Since low-speed streaks exist at the center of paired stream-wise vortices, span-wise spacing of the low-speed streaks can be evaluated by image processing. It must be reminded that the flake concentration is almost uniform anywhere including outside of the boundary layer. Thus, individual dots visible in the image do not express the simple presence of the flake but they inform the structure of liquid phase with the number density of the dots. 
a
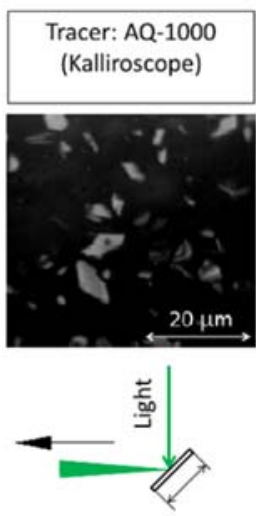

b

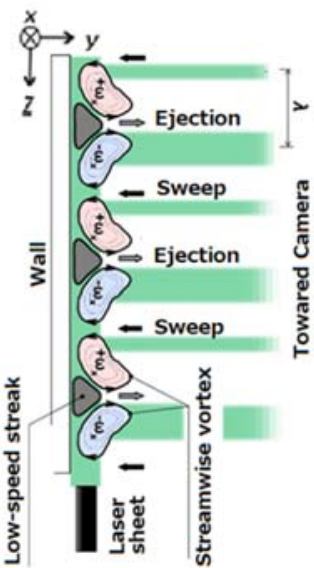

C

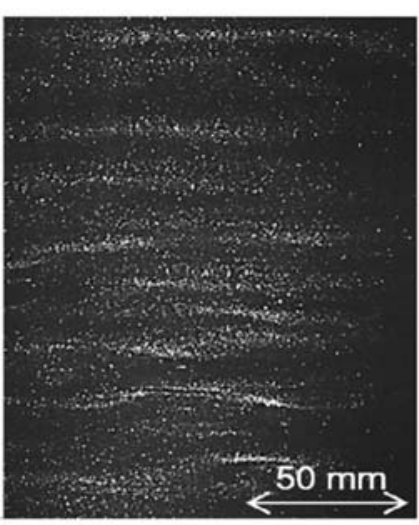

Fig. 4 Visualization of turbulent coherent structure using flake; (a) microscope image of Kalliroscople taken by the authors, (b) laser reflection pattern and (c) a sample snapshot in abscent of microbubbles

\section{3-2. Quantitative analysis}

Fig. 5 depicts time-line blow up image of the flake reflection at $\operatorname{Re}_{x}=2.00 \times 10^{4}(x=281 \mathrm{~mm})$. It is sampled from high-speed video camera movie on a span-wise segment $(1 \times 1024$ pixels $)$ at the center of the frame. Abscissa and ordinate indicate the time, and the span-wise coordinate, i.e. each panel expresses spatiotemporal distribution of stream-wise vortices. Known spacing of the low-speed streaks in single phase boundary layer is about $200 l_{\tau}$, which coincides with doubled spacing of the visible stripe in the figures, i.e. a pair of stream-wise vortices corresponds to a single low-speed streak between them. To measure these spacing, the time-line images are binarized by a threshold considering averaged brightness, and low-pass filtered by a two-dimensional median filter whose radius is approximately $9 l_{\tau}(10$ pixels). The binarized images are presented in Fig. $\mathbf{5 c}$ and $\mathbf{5 d}$, in which white blobs indicate stream-wise vortices extracted. 


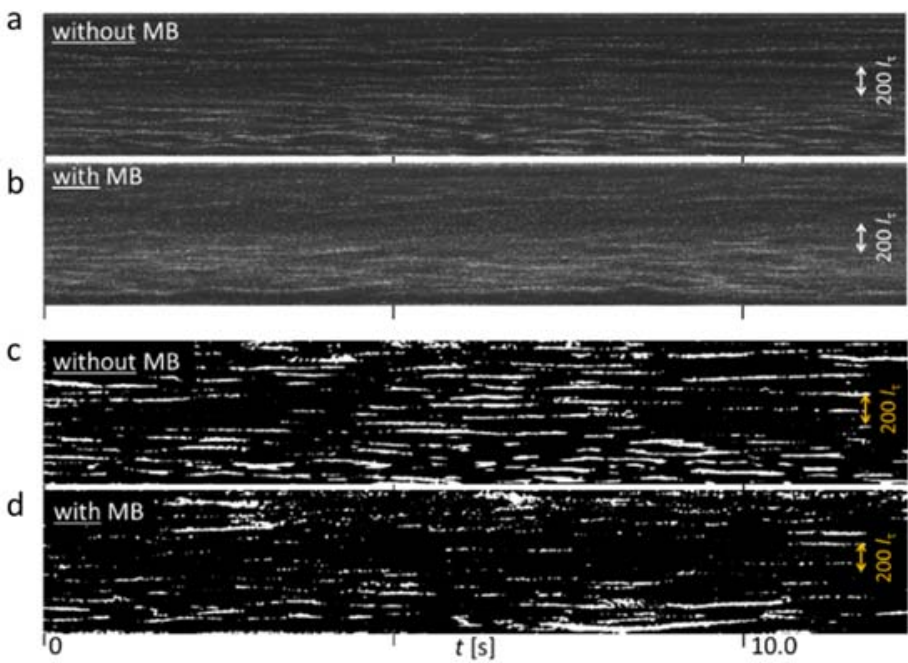

Fig. 5 Time-line blow up image of near-wall turbulent eddies visualized by micro grossy flake; (a) original image in the case without microbubble injection, (b) that with microbubble injection, (c) binarized image in the case without microbubble injection and (d) that with microbubble injection.

Fig. 6 shows how the spacing of low-speed streaks is measured by image processing. In the figure, the spanwise coordinate is normalized by the wall unit, $z^{+}=z / l_{\tau}$. A gray level profile along the red line in Fig. 6a is shown in Fig. 6b which involves many spiky peaks coming out due to light scattering of individual flakes, and most of them are much narrower than $40 l_{\tau}$. Since such a small eddy does not exist in theory, we convert the profile using a threshold to Fig. 6c in which individual locations of streamwise vortices and low-speed streaks become identifiable. 

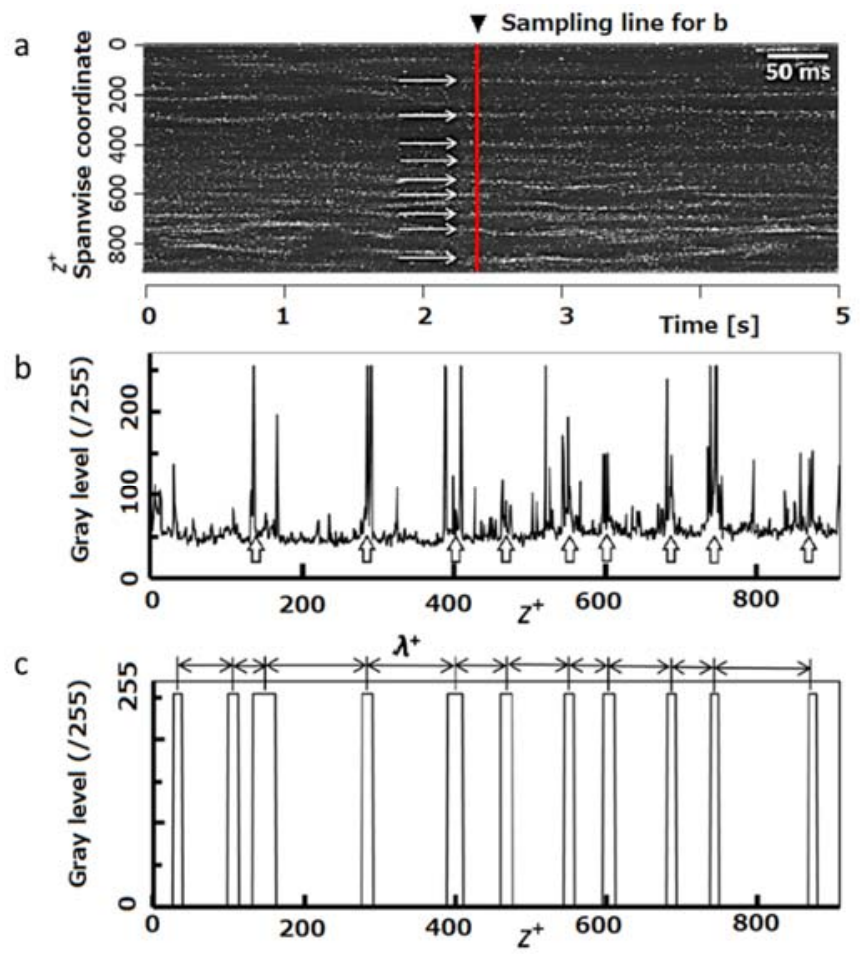

1

Fig. 6 Brightness profile of turbulent eddies visualized by flake; (a) time-line blow up image, (b) instantaneous brightness profile and (c) binarized profile of the brightness to extract locations of streamwise vortices and low-speed streaks

From the binarized profile, local interval of the low-speed streaks $\lambda^{+}$is measured for all the sampling period of 19 s using 9500 frames, and counted as a relative frequency histogram as shown in Fig. 7. The peak within the range of $50 \leq \lambda^{+}<60$ in the case without microbubble (Fig. 7a) corresponds to the half value of the mean spacing between two low-speed streaks and agrees with past studies $[29,30]$. In contrast, the histogram with microbubble additive takes a blunt peak in the range of $70 \leq \lambda^{+}<80$, indicating span-wise elongation of coherent structures. Here, the near-zero peak detected at $0 \leq \lambda^{+}<10$ (the red bar in Fig. 7b) comes from the noise caused by dot-wise scattering of laser light on microbubbles, irrelevantly to coherent structures, and can be ignored. Average value of the spacing $\overline{\lambda^{+}}$is obtained to be 107 in the case without microbubbles, and that with microbubbles is measured to be 132 . The increase means a structural elongation of near wall turbulence at $23.4 \%$, and infers with Eq. (8) that the wall friction is reduced by $34.3 \%$. 

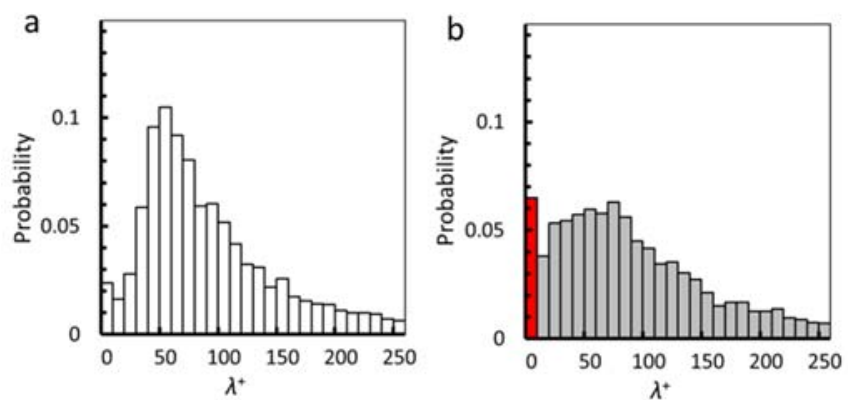

Fig. 7 Spanwise spacing of turbulent eddies measured from micro glossy flake visualization; (a) turbulent boundary layer without microbubbles and (b) the one with microbubble additive.

Finally, Fig. 8 represents measured relationship between visible lifetime of stream-wise vortices, $T$, and tilt angle of their principal axis from main flow direction, $\theta\left(-90^{\circ} \leq \theta \leq 90^{\circ}\right)$. Comparing the data between the two cases, occurrence frequency of stream-wise vortices is reduced with adding microbubbles while eddies with short code length survive. This trend qualitatively agrees with past studies $[34,35]$ which found collapse of turbulent coherent structures and reduction of Reynolds shear stress due to mixture of microbubbles.
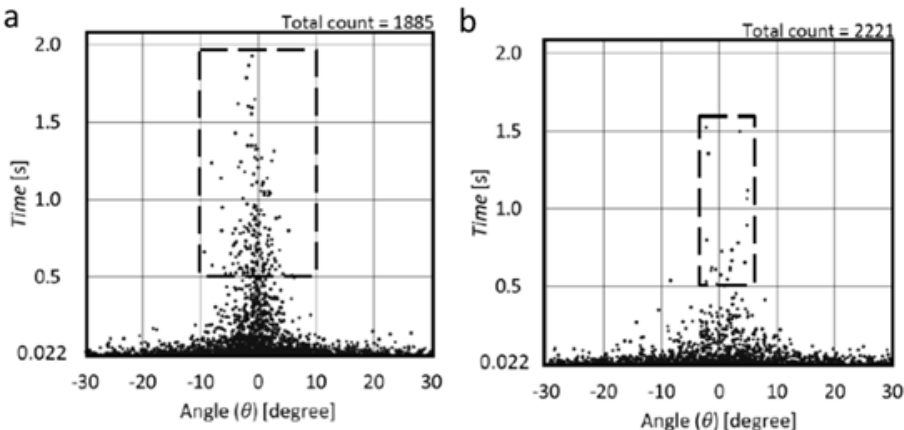

Fig. 8 Visible life time and tilting angle of streamwise vortices; (a) without microbubbles and (b) with microbubbles

\section{Rainbow illumination of microbubble clouds}

Considering of size of the turbulent vortices, almost $50 l_{\tau}$, this indicates that individual microbubbles cannot create liquid flow in their spatial scales to destroy turbulent vortices. Instead, formation of microbubble clouds much larger than the wall unit can alter the coherent structures in inertia region. Particles and bubbles in the turbulence are affected by the turbulence eddies and their spatial distribution is modified [36-38]. In this section, we introduce a method for visualizing microbubble clouds by rainbow illumination and investigate spatial distribution of microbubbles in the boundary layer. Basic idea of rainbow illumination is similar to color-coded 3-D particle tracking velocimetry for identifying tracer particles [24]. In such a PTV, 
the out-of-plane coordinate of individual particle is estimated from hue value of the colored particles to be combined with two in-plane coordinates. In application to microbubbles' coloring, difference arises due to unique optical characteristics of spherical gas-liquid interface as explained below.

\section{4-1. Hue characteristics of scattering liqht from microbubbles}

Natural rainbow in atmosphere emerges due to spherical water droplets scattering light in slightly different direction dependent on wavelength of the light. In cases of microbubbles in water, similar phenomenon occurs in white lighting, which leaves pseudo color in image plane. In advance of the main experiment, how microbubbles scatter color-coded light in a quiescent water is investigated using a simple experiment shown in Fig. 9. Schematic diagram of the experiment is presented in Fig. 9a. The illuminated color pattern is controlled by a PC connected to the liquid crystal display projector. In this examination, color is varied in the span-wise direction of a vertical plate in the water container. A high-speed camera is located outside the container at $110^{\circ}$ to the plate surface, according to the brightest illumination angle of spherical bubbles. A snapshot recorded by the camera is shown in Fig. 9b. By thresholding the brightness value, individual position of microbubbles and their hue values are obtained as plotted in Fig. 9c. Open circles represent measured hue values with a standard deviation at each position. A red curve in the graph indicates moving averaged value of the measured data.

Ideally, the red curve becomes a linear; illuminated hue values coincide with measured hue values. Displacement from the linearity originates from two factors. One is microbubble's optical scattering characteristics dependent on the scattering direction analogous to rainbow principle. Another is lightreceiving characteristic of the camera, which relies on a type of image sensors receiving multi-spectral light for three primary color bands. This usually brings hue-shift to the three primary colors (red at $0^{\circ}$, green at $120^{\circ}$, blue at $240^{\circ}$ ). In addition, there are further minor factors influencing the relationship, such as mutual light scattering among microbubbles, light attenuation due to overlap of microbubbles in imaging plane, and digital image compression which does not guarantee conservation of hue value. Regardless to these influences, the moving averaged curve takes a monotonic function with increase in illuminated hue values. This allows us to correct measured hue values uniquely and recursively to the original value, and thus, to determine the position of the microbubbles. For the recursive approach, systematic and random errors are estimated from local flatness of the moving-averaged curve and local deviation of the plots, respectively. From the data, maximum error is estimated to be $20^{\circ}$ for the both components. This indicates that we classify whole the domain into $18\left(=360^{\circ} / 20^{\circ}\right)$ layers in the depth direction. 
a

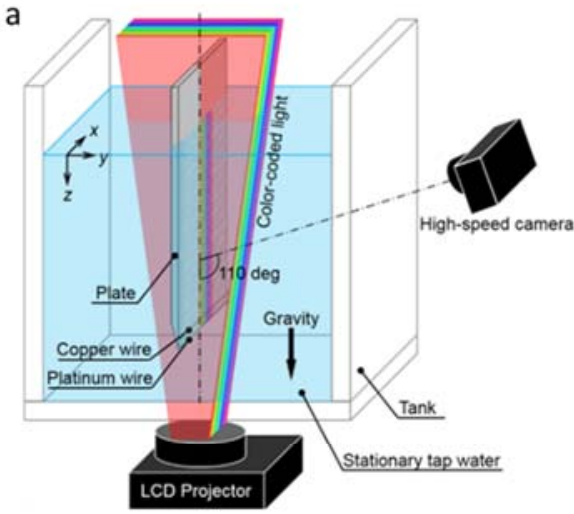

b

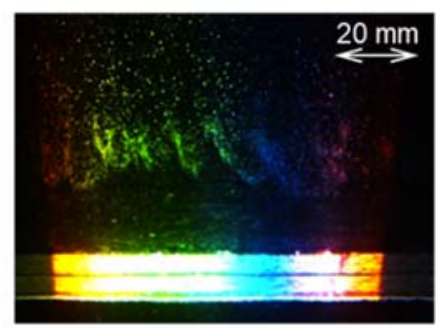

c

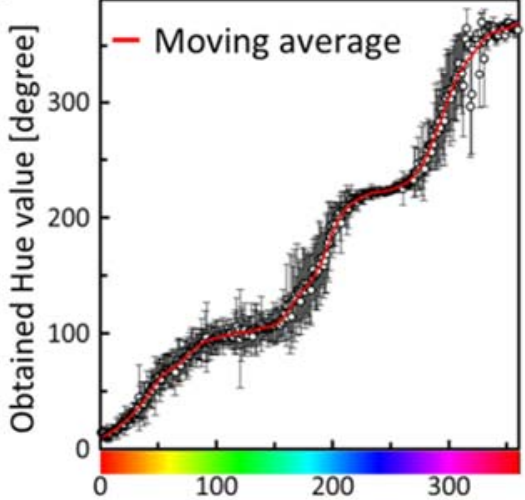

Illuminated Hue value [degree]

Fig. 9 A color scattering test of microbubbles; (a) experimental set-up for a color scattering test, (b) a snapshot image of hydrogen microbubbles and (c) relationship between illuminated hue and measured hue values

\section{4-2. Volumetric illumination of boundary layer}

Learning from the examination, rainbow illumination is applied to the turbulent boundary layer along the test plate in the open channel as illustrated in Fig. 10a. Hue value is changed linearly to the distance from the plate surface on the order of red, magenta, blue, cyan, green, yellow and returning to red. Width of the rainbow part is set $12 \mathrm{~mm}$, comparable to the boundary layer thickness. Red monochromatic light is widely projected in the outer layer at $y>12 \mathrm{~mm}\left(\right.$ about $\left.y^{+}>55\right)$ so that microbubbles bursting off the boundary layer is identified as red color.

An original snapshot of microbubbles image is shown in Fig. 10b. The image is regulated dark purposely in order to avoid color saturation of image sensors, which has maximum brightness lower than 100 relative to 255 brightness level. For easier look in printed manuscript, brightness amplified image of the same snapshot is shown in Fig. 10c. Cyan dots in the image correspond to microbubbles suspended in wall proximity at $y<3 \mathrm{~mm}$ (about $y^{+}<13$ ), while magenta dots are microbubbles accumulated in turbulent eddies at $4<y<7 \mathrm{~mm}$ (about $18<y^{+}<32$ ). Yellow spots are microbubbles being ejected toward the outer layer at $9<y<12 \mathrm{~mm}$ (about $41<y^{+}<55$ ). It is noted that exact computation of the depth coordinate $y$ from the color image is applied for the original image with hue value conservation but not for the brighness amplified image. 

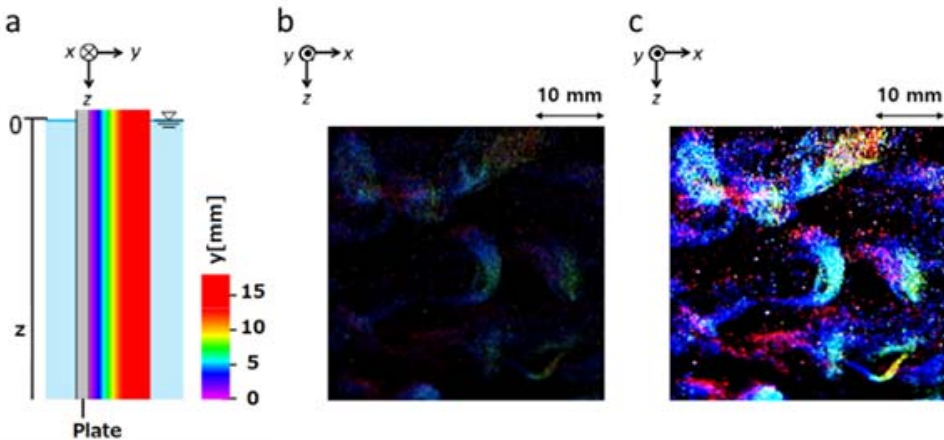

Fig. 10 Microbubble visualization by means of color-coded volumetric illumination; (a) tangential projection of color-coded light, (b) an original sample image of showing hair-pin shaped microbubble clouds and (c) its brightness amplified image

\section{4-3. Spatio-temporally resolved distribution}

By sampling a vertical segment from a movie taken by the high-speed video camera, time-line blow-up images are generated (Fig. 11). In each panel, the horizontal axis indicates time within 5 seconds. The scale added on the top is an estimated length scale in the stream-wise coordinate according to $x=U_{\mathrm{m}} t$, with which spatial wavelength of microbubble clouds is understood.
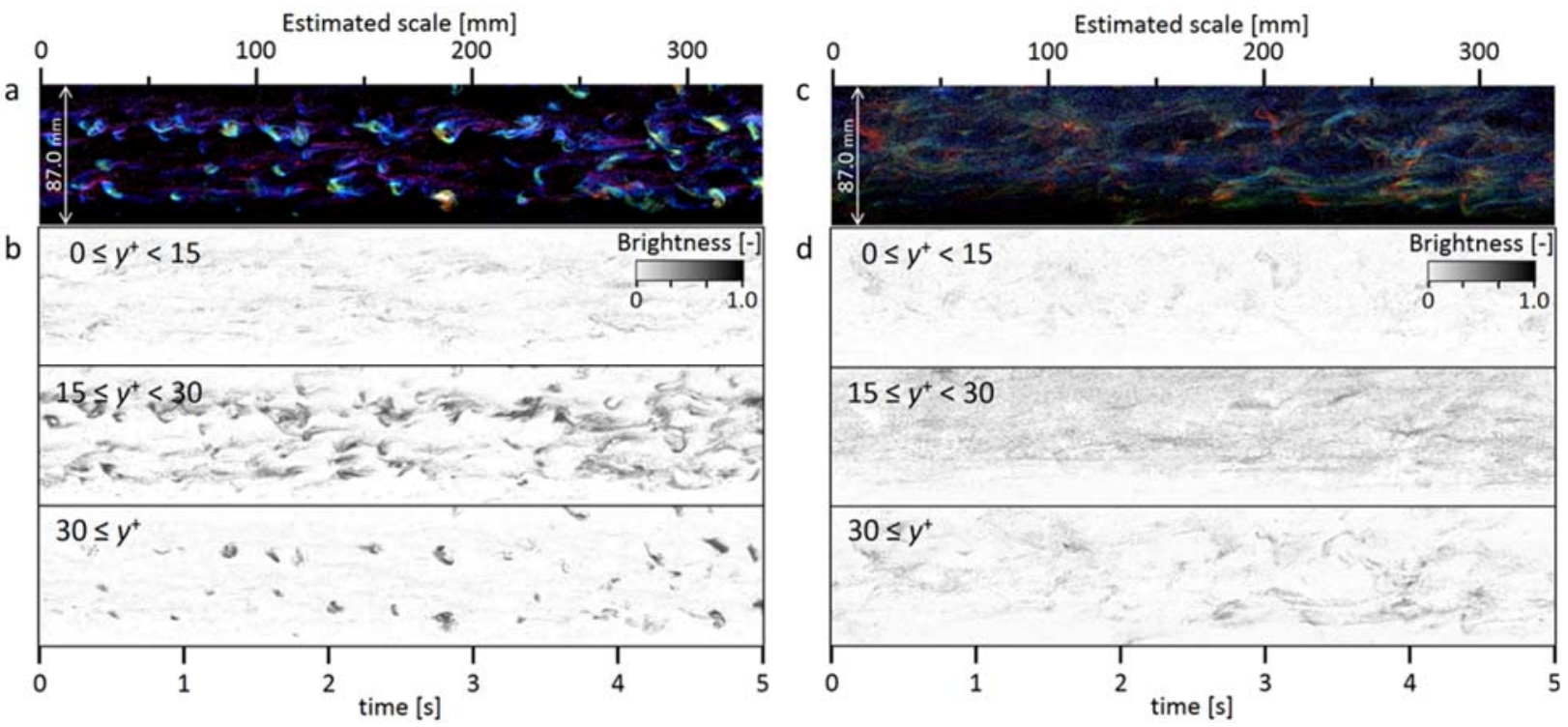

Fig. 11 Time-line blow up image of colored microbubbles; (a, c) a color image and (b, d) its separated images to each layer, where (a, b) $R e_{x}=9660$ and (c, d) $R e_{x}=22500$

A color image of microbubble distribution recorded at $R e_{x}=9660$ is shown in Fig. 11a. By converting the hue values to $y$ coordinate, the color time-line blow up images are separated into three layers: viscous sublayer $\left(0 \leq y^{+}<15\right)$, buffer layer $\left(15 \leq y^{+}<30\right)$ and logarithmic layer $\left(30 \leq y^{+}\right)$as shown in Fig. 11b, where 
wall coordinate is defined by $y^{+}=y / l_{\tau}$. The brightness in each layer corresponds to local number density of the microbubbles. In the viscous sublayer, microbubbles are distributed with a weak stripe pattern. Span-wise spacing of the stripes coincides with that of low-speed streak at this Re number. Inside the buffer layer, microbubbles form localized clouds that have high number density than in the viscous sublayer. It is observable that large clouds pass by at a frequency of around $2 \mathrm{~Hz}$, corresponding to $50 \mathrm{~mm}\left(\sim 220 l_{\tau}\right)$ in stream-wise interval. In the logarithmic layer, microbubbles come out as spots. These spots are regarded as head of each cloud in the buffer layer, conveyed by ejection of liquid phase. In the downstream region at $R e_{x}$ $=22500$ (see Fig. 11c to Fig. 11d), these localized clouds are relatively dispersed all over the layer but most of the microbubbles still stay inside each layer. Difference of the cloud formation between the two positions is explained by that of enstrophy in liquid phase. That is, liquid phase induces high shear rate in the upstream position to rapidly capture the microbubbles in hair-pin shaped vortex cores. To the contrary, the shear rate decreases in the downstream region with expanded boundary layer thickness, resulting in calmed cloud formation.

a

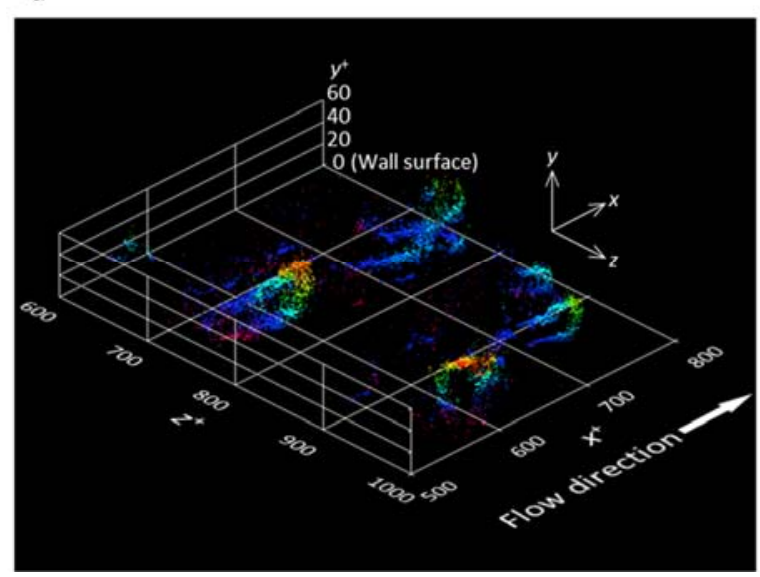

b

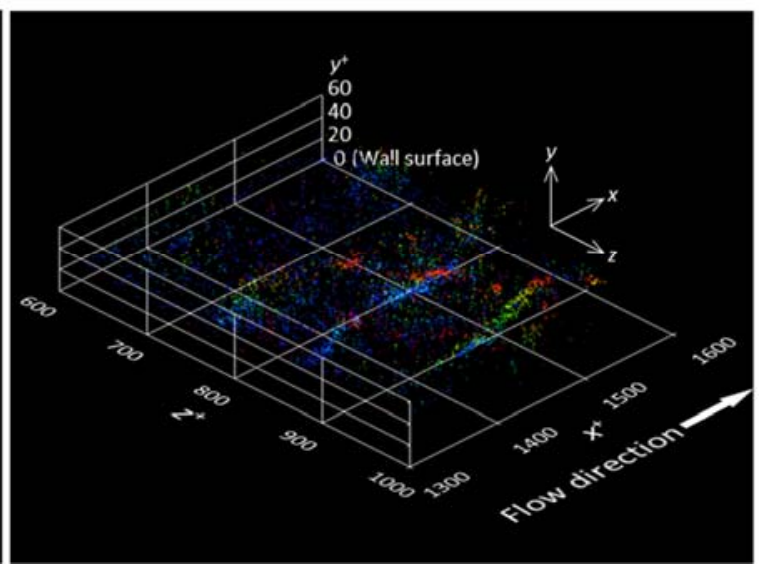

Fig. 12 3-D depiction of microbubble distribution from hue-to-depth coordinate transform; (a) upstream position around $R e_{\mathrm{x}}=9660$ and (b) downstream position around $R e_{\mathrm{x}}=22500$

By the hue-to-depth conversion, 3-D distributions of microbubbles are depicted in Fig. 12 using the 3D coordinate normalized by wall unit. Color indicates the individual position of microbubbles from the wall. At $R e_{x}=9660$, each cloud has hair-pin shapes stretched in the stream-wise direction. Coherent structures in turbulent boundary layer such as ejection, sweep, and low-speed streaks induce these cloud formations. Ejection plays a primary role because of the following sequence: ejection collects microbubbles suspended in viscous sublayer and conveys them along outward flow to logarithmic layer. In fact, we often found the microbubble clouds like in Fig. 13. In hair-pin shaped cloud (Fig, 13a), local number density of microbubbles is estimated to be higher than $10^{4} / \mathrm{cm}^{3}$, and its volume fraction gets close to the packing limit of spheres. In the logarithmic layer, clam-shell shaped clouds often appeared. This infers a long Lagrangian interaction 

in these highly dense clouds, of which reason is attributed to rigidity of microbubbles due to strong surface tension [15] and also to local repulsion generated by Zeta potential on the microbubble surface in water [39$42]$.

a

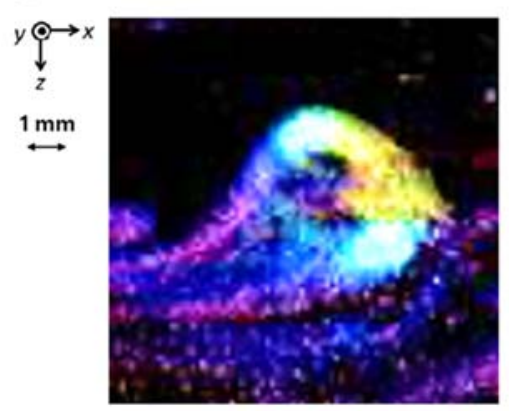

b

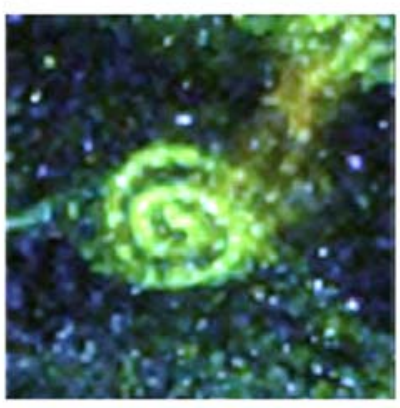

Fig. 13 Microbubble clouds observed at $R e_{x}=9660$; (a) a hair-pin shaped cloud rising up to the buffer layer having legs in viscous sublayer and (b) clam shell shaped cloud ejected to logarithmic layer, inferring long Lagrangian interaction between microbubbles and individual eddies

\section{4-4. Averaged concentration profiles}

To evaluate bulk turbulent diffusivity of the microbubbles, time-averaged concentration profile is measured.

Fig. 14 represents the cumulative histograms of microbubble clouds, which is a bubble-presense probability integrated in $y$ direction. The value takes 0 at $y=0$, and unity at infinity far from the wall. Circle and trianglar symbols in the graph are measured from the rainbow visualization at two different $R e_{x}$ numbers. Cross symbols are the data measured by a single laser-sheet illumination of microbubbles with traversing the $y$ coordinate (see Fig. 3a). 


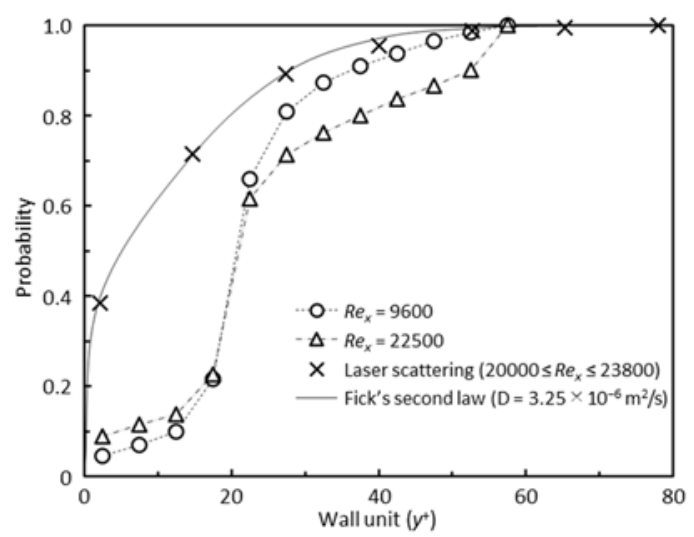

Fig. 14 Cumulative frequency histogram of microbubble concentration profile inside the turbulent boundary layer measured by color-coded illumination and laser scattering methods.

The curve fitting the cross symbols is the value according to Fick's second law assuming a constant diffusivity of microbubbles as the simplest model. The curve is obtained using the following one-dimensional diffusion equation for a concentration profile;

$$
\frac{\partial C}{\partial t}=D \frac{\partial^{2} C}{\partial y^{2}}
$$

where $C(y, t)$ is the concentration of microbubbles, and $D$ denotes the microbubble diffusivity with a dimension of $\mathrm{m}^{2} / \mathrm{s}$. As giving $C(y, 0)$ a delta function at $y=0$, Eq. (9) gives universal solution as

$$
C(y, t)=\frac{1}{2 \sqrt{\pi D t}} \exp \left(-\frac{y^{2}}{4 D t}\right) .
$$

Substituting present experimental conditions such as $t=x / U_{\mathrm{m}}$ into Eq. (10), the most matched profile with experimental data is derived by changing the diffusivity $D$. From least square approach, we have obtained the microbubble diffusivity as $D=3.2 \times 10^{-6} \mathrm{~m}^{2} / \mathrm{s}$. This value is larger than kinematic viscosity of water, $v=$ $0.9 \times 10^{-6} \mathrm{~m}^{2} / \mathrm{s}$ in present experiment, indicating active diffusion of microbubbles more than molecular momentum diffusivity of water. Effective Schmidt number of microbubbles is hence estimated as $S c=v / D=$ 0.31 . In contrast, turbulent momentum diffusivity is estimated as $v_{\mathrm{t}}=l_{\tau} U_{\mathrm{m}}=1.49 \times 10^{-5} \mathrm{~m}^{2} / \mathrm{s}$ on the present liquid flow condition. Thus, the relative diffusivity of microbubbles is $D / v_{\mathrm{t}}=0.21$, being sufficiently smaller than unity. This tells that microbubbles tend to stay inside the turbulent boundary layer relatively to the expansion of the boundary layer thickness in $y$ direction. The trend can be also confirmed in Fig. 14 that $90 \%$ of microbubbles are concentrated inside the wall-closest layer of $0<y^{+}<30$. Such reduced diffusion effect of microbubbles against turbulence is attributed to cloud formation inside the buffer layer where microbubbles are captured into vortex cores. Accumulation and diffusion of small dispersion in turbulence is generally explained by crossing trajectories of the dispersion to individual eddies [36, 43, 44]. It is indisputable that primary force to accumulate microbubbles into vortex cores is local pressure gradient, i.e. 
high enstrophy inside the vortices. Lift force of spherical microbubbles with small vertical slip also assists their accumulation; here lift acts in Saffman regime because of $R e_{\mathrm{b}}<1$, and orients toward the wall.

Another point found from the data is the difference of the profile between laser scattering measurement and rainbow illumination. The rainbow illumination underestimates the cumulative histogram at $0<y^{+}<60$. This is due to optical occlusion of microbubbles as microbubbles form high-density clouds. Microbubbles existing under microbubble clouds cannot be counted by the rainbow illumination. This is regarded as disadvantage of the rainbow illumination, however, it also infers that the gap between two measurements proves generation of microbubble clouds in the corresponding layer. Since the cumulative histogram steeply recovers at $20<y^{+}<30$, it is understood that dense microbubble clouds are actively induced mainly in the buffer layer. This fact is reasonable since enstrophy takes the highest value inside the buffer layer to produce slip velocity between two phases.

\section{Discussions}

Why can microbubbles alter the coherent structures of wall turbulence so sensitively? Only at $0.01 \%$ of microbubble volume fraction given to the boundary layer, we confirmed visible modification of the inner layer structure leading to $34 \%$ of drag reduction. Note that this drag reduction is not directly measured value and just estimated value from the increasing average space between streaks with microbubbles. Similar impact at $0.03 \%$ of microbubble volume fraction was reported by Hara et al. [23] who confirmed $30 \%$ of drag reduction. They conducted PTV measurement and found local Reynolds shear stress reduced only inside the buffer layer. For volume fraction larger than $0.2 \%$, Serizawa et al. [45] and Shattet et al. [22] reported relaminarization of pipe flows as viewed from measured pipe friction factors in a turbulent flow transition regime. At further higher volume fraction over $2 \%$, volumetric impact of microbubbles starts to dominate as reported by Ferrante and Elghobashi [46] using DNS and Zhen and Hassan [20] by PIV. Back to the first question, we attempt to derive the most possible reason which survives at dilute limit of microbubbles oneby-one as below.

\section{5-1. High interfacial concentration}

Even though volume fraction of microbubble is controlled low, total area of gas-liquid interface is provided very high. To quantify this effect, following quantity can represent dimensionless interfacial concentration as a mean value inside the boundary layer:

$$
\beta=\pi d^{2} \frac{N}{W L}=\frac{6 \delta}{d} \alpha, \because \alpha=\frac{N}{W L \delta} \frac{\pi d^{3}}{6},
$$

where $\alpha$ is void fraction inside the boundary layer defined by the span-wise length $W$, the stream-wise length $L$, and the boundary layer thickness $\delta$. Here, $d$ and $N$ stand for the microbubble diameter and the number of 
microbubbles in the volume of $L W \delta$. The quantity $\beta$ indicates the ratio of total microbubble interfacial area inside boundary layer to the area of wall surface. In the present flow condition, Eq. (11) becomes $\beta=2400 \alpha$ at $\delta=12 \mathrm{~mm}$ and $d=30 \mu \mathrm{m}$. Thus, $\alpha=0.01 \%$ provides $\beta=24 \%$. This is superficially equivalent to the wall occupied by gas film at $24 \%$. Since momentum exchange between two phases in Stokes drag regime increases proportionally to the interfacial area concentration, the parameter could be an appropriate factor to explain the magnitude of the microbubbles' impact on the modification of coherent structures in the turbulent boundary layer.

\section{5-2. Buoyancy in localized state}

Buoyant force of microbubbles acts on liquid flow in proportion to the microbubble volume fraction. Impact of the buoyant force to the liquid flow can be estimated by the ratio of buoyant potential $E_{\mathrm{b}}$ to kinetic energy of liquid flow $E_{1}$ inside the boundary layer as

$$
\varepsilon=\frac{E_{\mathrm{b}}}{E_{1}}=\frac{N \rho g \pi d^{3} / 6}{W \int_{0}^{\delta} 1 / 2 \rho \bar{u}^{2}(y) d y}=\frac{9 \alpha g \delta}{7 U_{\mathrm{m}}{ }^{2}},
$$

where primitive parameters in the definition are the same as previous ones. In the present flow condition, Eq. (12) becomes $\varepsilon=40 \alpha$, thus $\varepsilon=0.4 \%$ at $\alpha=0.01 \%$. This value is two-order smaller than $\beta$ estimated in former section, but is still significant. Actual impact of buoyancy may be intensified more than Eq. (12) with two reasons. One is generation of microbubble clouds taking much higher value of local volume fraction. The other is increase of buoyant action to liquid phase as spatial gradient of local volume fraction is given. These effects were experimentally confirmed in Taylor-Couette flows, where microbubbles are preferentially accumulated to selected locations of vortices [24]. Thus, if denominator of Eq. (12) is replaced with kinetic energy of the secondary mode such as primary vortex in shear, the impact becomes one-order higher to be comparable to drag reduction rate. In short, localization of microbubble distribution always calls intensified buoyant action to coherent structures regardless to Froude number defined in the bulk space.

\section{5-3. Microbubble cloud rheology}

As microbubbles form clouds that have high local number density inside, viscosity of the clouds increases drastically. Murai et al. [47] measured such effective viscosity with around $45 \%$ of void fractions using interfacial rheometry. Their result is approximated as

$$
\eta=\frac{\mu_{\mathrm{b}}}{\mu_{1}}=1300\left(\frac{C a}{10^{-8}}\right)^{-0.45}, C a=\frac{\mu_{1} d}{2 \sigma} \dot{\gamma},
$$

where $\eta$ is ratio of microbubble cloud viscosity $\mu_{\mathrm{b}}$ to liquid viscosity $\mu_{\mathrm{l}}$. Ca is capillary number for a single microbubble with diameter of $d$, interfacial tension of $\sigma$, and surrounding liquid shear rate of $\dot{\gamma}$. The formula is valid only for $10^{-8}<C a<10^{-5}$, and $35 \%<\alpha<55 \%$. Substituting the present flow conditions into the formula obtains $\mathrm{Ca}=1.5 \times 10^{-6}$, and $\eta=140$. For the range of $\alpha<35 \%$, the following empirical formula 
proposed by Mooney [48] is available,

$$
\eta=\exp \left(\frac{A \alpha}{1-k \alpha}\right), 1.35<k<1.91
$$

where $A$ and $k$ stand for dimensionless parameters describing the frictional impact and spatial arrangement pattern of spherical dispersion, respectively. Assuming $k=1.5$ as a random distribution, coupling of Eq. (13) and Eq. (14) gives $A=6.7$. Thus, the viscosity ratio takes $\eta=38$ at $\alpha=30 \%, \eta=6.7$ at $\alpha=20 \%$, and $\eta=2.2$ at $\alpha=10 \%$. These values are obviously to explain that microbubble clouds strongly resist to deformation of fluid. Moreover, we have confirmed that microbubble clouds were induced in vortex cores. This implies the rheological effect which acts mainly to decay of turbulence.

\section{Conclusions}

In this paper, we reported how microbubbles interact with eddies in a spatially developing turbulent boundary layer along a flat wall. We have adapted a rainbow visualization technique to explore microbubbles' preferential concentration into low-speed streak regions close to the viscous sublayer and into hair-pin shaped clouds in the buffer layer. These accumulations provided reduced diffusion effect of microbubbles as proved by microbubble Schmidt number that was obtained smaller than unity. We also visualized turbulent eddies in the liquid phase with flake optics, and confirmed a significant elongation of span-wise spacing of low-speed streaks and also a reduction of occurrence frequency of long stream-wise vortices. In the final part, we tried to offer the reason why only $0.01 \%$ of microbubble volume fraction can cause clearly visible alternation of the coherent structure. It is mainly attributed to effect of microbubble clouds on interfacial concentration, localized buoyancy, and effective viscosity. While some of them still need further experimental proving, we expect that the present experimental results re-accelerate the study of microbubble drag reduction.

\section{Acknowledgments}

The work presented in the manuscript was supported by JSPS KAKENHI grant nos. 17H01245 and 17K14583, and Innovative Science \& Technology Initiative for Security, ATLA, Japan. Also the authors express thanks to Mr. Toshiyuki Sampo, Dr. Yoshiaki Takahashi.

\section{References}

[1] M. McCormick, R. Bhattacharyya, Drag reduction of a submersible hull by electrolysis, Nav. Eng. J. 85 (1973) 11-16.

[2] H.H. Legner, Simple model for gas bubble drag reduction, Phys. Fluids 27 (1984) 2788-2790.

[3] J.L. Marie, A simple analytical formulation for microbubble drag reduction, PhysicoChemical Hydrodynamics 8 (1987) 213-220. 
1 [4] C.L. Merkle, S. Deutsch, Drag reduction in liquid boundary layers by gas injection, Prog. Astronaut. Aeronaut. 123 (1990) 351-411.

[5] C.L. Merkle, S. Deutsch, Microbubble drag reduction in liquid turbulent boundary layers, ASME Appl. Mech. Rev. 45 (1992) 103-127.

[6] H. Kato, T. Iwashina, M. Miyanaga, H. Yamaguchi, Effect of microbubbles on the structure of turbulence in a turbulent boundary layer, J. Mar. Sci. Tech. 4 (1999) 115-162.

[7] Y. Kodama, A. Kakugawa, T. Takahashi, H. Kawashima, Experimental study on microbubbles and their applicability to ships for skin friction reduction, Int. J. Heat Fluid Flow, 21 (2000) 582-588.

[8] Y. Moriguchi, H. Kato, Influence of microbubble diameter and distribution on frictional resistance reduction, J. Mar. Sci. Tech. 7 (2002) 79-85.

[9] A. Kitagawa, K. Hishida, Y. Kodama, Flow structure of microbubble-laden turbulent channel flow measured by PIV combined with the shadow image technique, Exp. Fluids 38 (2005) 466-475.

[10] Y. Tasaka, T. Kimura, Y. Murai, Estimating the effective viscosity of bubble suspensions in oscillatory shear flows by means of ultrasonic spinning rheometry, Exp. Fluids 56 (2015) 1867.

[11] D.P.M. van Gils, D.N. Guzman, C. Sun, D. Lohse, The importance of bubble deformability for strong drag reduction in bubbly turbulent Taylor-Couette flow, J. Fluids Mech. 722 (2013) 317-347.

[12] H.J. Park, Y. Tasaka, Y. Murai, Bubbly drag reduction investigated by time-resolved ultrasonic pulse echography for liquid films creeping inside a turbulent boundary layer, Exp. Therm. Fluid Sci. 103 (2019) 66-77.

[13] A. Kitagawa, P. Denissenko, Y. Murai, Behavior of bubbles moving along horizontal flat plates with different surface wettability, Exp. Therm. Fluid Sci. 104 (2019) 141-152.

[14] A. Ferrante, S. Elghobashi, On the physical mechanism of drag reduction in a spatially developing turbulent boundary layer laden with microbubbles, J. Fluid Mech. 503 (2004) 345-355.

[15] Y. Murai, T. Shiratori, I. Kumagai, P. A. Ruehs, P. Fischer, Effective viscosity measurement of interfacial bubble and particle layers at high volume fraction, Flow Meas. Instrum. 41 (2016) 121-128.

[16] Y. Tasaka, Y. Yoshida, R. Rapberger, Y. Murai, Linear viscoelastic analysis using frequency-domain algorithm on oscillating circular shear flows for bubble suspensions, Rheologica Acta 57 (2018) 229240.

[17] T. Watamura, A. Kitagawa, Y. Murai, Cloud clustering of microbubbles ascending along a vertical wall proximity, Chem. Eng. Sci. 208 (2019) 115132.

[18] V. Mathai, E. Calzavarini, J. Brons, C. Sun, D. Lohse, Microbubbles and microparticles are not faithful tracers of turbulent acceleration. Phys. Rev. Lett. 117 (2016) 024501. 
[19] Y. Murai, Y. Oishi, Y. Takeda, F. Yamamoto, Turbulent shear stress profiles in a bubbly channel flow assessed by particle tracking velocimetry, Exp. Fluids 41 (2006) 343-352.

[20] L. Zhen, Y.A. Hassan, Wavelet autocorrelation identification of the turbulent flow multi-scales for drag reduction process in microbubbly flows, Chem. Eng. Sci. 61 (2006) 7107-7114.

[21] Y. Murai, H. Oiwa, Y. Takeda, Frictional drag reduction in bubbly Couette-Taylor flow, Phys. Fluids 20 (2008) 034101.

[22] M.M.E. Shatat, S. Yanase, T. Takami, T. Hyakutake, Drag Reduction Effects of Micro-Bubbles in Straight and Helical Pipes, J. Fluid Sci. Tech. 4 (2009) 156-167.

[23] K. Hara, T. Suzuki, F. Yamamoto, Image analysis applied to study on frictional drag reduction by electrolytic microbubbles in a turbulent channel flow, Exp. Fluids 50 (2011) 715-727.

[24] T. Watamura, Y. Tasaka, Y. Murai, Intensified and attenuated waves in a microbubble Taylor-Couette flow, Phys. Fluids 25 (2013) 054107.

[25]J. Xiong, R. Idoughi, A.A. Aguirre-Pablo, A.B. Aljedaani, X. Dun, Q. Fu, S.T. Thoroddsen, W. Heidrich, Rainbow particle imaging velocimetry for dense 3D fluid velocity imaging, ACM Trans. Graph. 36 (2017) 36 .

[26] P. Matisse, M. Gorman, Neutrally buoyant anisotropic particles for flow visualization, Phys. Fluids 27 (1984) 759.

[27] J.Ohkubo, Y. Tasaka, H.J. Park, Y. Murai, Extraction of 3D vortex structures from a turbulent puff in a pipe using two-color illumination and flakes, J. vis. 19 (2016) 643-651.

[28] W. Cheng, Y. Murai, T. Sasaki, F. Yamamoto, Bubble velocity measurement with a recursive cross correlation PIV technique, Flow Meas. Instrum. 16 (2005) 35-46.

[29] C.R. Smith, S.P. Metzler, The characteristics of low-speed streaks in the near-wall region of a turbulent boundary layer, J. Fluid Mech. 129 (1983) 27-54.

[30] M. Zacksenhouse, G. Abramovich, G. Hetsroni, Automatic spatial characterization of low-speed streaks from thermal images, Exp. Fluids 31 (2001) 229-239.

[31] E.K. Longmire, I. Marusic, S. Pothos, Dual-plane PIV technique to determine the complete velocity gradient tensor in a turbulent boundary layer, Exp. Fluids 39 (2005) 222-231.

[32] S. Robinson, Coherent Motions in the turbulent boundary layer, Annu. Rev. Fluid Mech. 23 (1991) 601639.

[33] H.J. Park, Y. Tasaka, Y. Murai, Vortical structures swept by a bubble swarm in turbulent boundary layers, Chem. Eng. Sci. 116 (2014) 486-496.

[34] Y. Oishi, Y. Murai, Y. Tasaka, Y. Takeda, Motion of Microbubbles Relative to Streamwise Vortices in 
the Wall Turbulence, 6th Int. Conf. Multiphase Flow (2007) 535.

[35] C.C. Gutierrez-Torres, Y.A. Hassan, J.A. Jimenez-Bernal, Turbulence structure modification and drag reduction by microbubble injections in a boundary layer channel flow, J. Fluids Eng. 130 (2008) 111304.

[36] M. R. Maxey, The gravitational setting of aerosol particles in homogeneous turbulence and random flow fields, J. Fluid Mech. 174 (1987) 441-645.

[37] H. Parishani, O. Ayala, B. Rosa, L.-P. Wang, W.W. Grabowski, Effects of gravity on the acceleration and pair statistics of inertial particles in homogeneous isotropic turbulence, Phys. Fluids 27 (2015) 033304.

[38] V. Mathai, S.G. Huisman, C. Sun, D. Lohse, M. Bourgoin, Dispersion of air bubbles in isotropic turbulence, Phys. Rev. Lett. 121 (2018) 054501.

[39] S. Usui, H. Sasaki, H. Matsukawa, The dependence of Zeta potential on bubble size as determined by the Dorn effect, J. Colloid Interface Sci. 81 (1981) 80-84.

[40] R.H. Yoon, J.L. Yordan, Zeta potential measurements on microbubbles generated using various surfactants, J. Colloid Interface Sci. 113 (1986) 430-438.

[41] M. Takahashi, Zeta potential of microbubbles in aqueous solutions - electrical properties of the gasliquid interface, J. Phys. Chem. 109 (2005) 21858-21864.

[42] F.Y. Ushikubo, M. Enari, T. Furukawa, R. Nakagawa, Y. Makino, Y. Kawagoe, S. Oshita, Zeta-potential of Micro- and/or Nano-bubbles in Water Produced by Some Kinds of Gases, IFAC Proc. Vol. 43 (2010) 283-288.

[43] F. Risso, Agitation, Mixing, and Transfers Induced by Bubbles, Annu. Rev. Fluid Mech. 50 (2018) 2548.

[44] E. Alméras, V. Mathai, C. Sun, D. Lohse, Mixing induced by a bubble swarm rising through incident turbulence, Int. J. Multiphase Flow 114 (2019) 316-322.

[45] A. Serizawa, T. Inui, T. Eguchi, Flow characteristics and pseudo-laminarization of vertically upward air-water milky bubbly flow with micro bubbles in a pipe, Jpn. J. Multiphase Flow 19 (2005) 335-340 (in Japanese).

[46] A. Ferrante, S. Elghobashi, Reynolds number effect on drag reduction in a microbubble- laden spatially developing turbulent boundary layer, J. Fluid Mech. 543 (2005) 93-106.

[47] Y. Murai, T. Shiratori, I. Kumagai, P.A. Ruehs, P. Fischer, Effective viscosity measurement of interfacial bubble and particle layers at high volume fraction, Flow Meas. Instrum. 41 (2015) 121-128.

[48] M. Mooney, The viscosity of a concentrated suspension of spherical particles, J. Colloid Sci. 6 (1951) $162-170$. 